\title{
ALDH1A1 expression correlates with clinicopathologic features and poor prognosis of breast cancer patients: a systematic review and meta-analysis
}

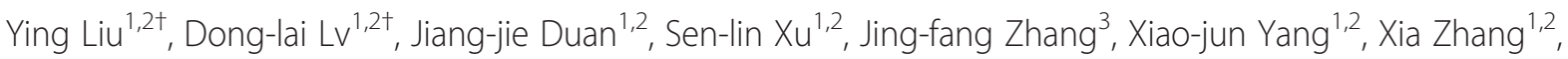
You-hong Cui ${ }^{1,2}$, Xiu-wu Bian ${ }^{1,2^{*}}$ and Shi-cang Yu ${ }^{1,2^{*}}$

\begin{abstract}
Background: Aldehyde dehydrogenase 1 family member A1 (ALDH1A1) has been identified as a putative cancer stem cell (CSC) marker in breast cancer. However, the clinicopathological and prognostic significance of this protein in breast cancer patients remains controversial.

Methods: This meta-analysis was conducted to address the above issues using 15 publications covering 921 $\mathrm{ALDH} 1 \mathrm{~A} 1^{+}$cases and 2353 controls. The overall and subcategory analyses were performed to detect the association between ALDH1A1 expression and clinicopathological/prognostic parameters in breast cancer patients.

Results: The overall analysis showed that higher expression of ALDH1A1 is associated with larger tumor size, higher histological grade, greater possibility of lymph node metastasis (LNM), higher level expression of epidermal growth factor receptor 2 (HER2), and lower level expression of estrogen receptor (ER)/progesterone receptor (PR). The prognosis of breast cancer patients with $\mathrm{ALDH1A} 1^{+}$tumors was poorer than that of the $\mathrm{ALDH} 1 \mathrm{~A} 1^{-}$patients. Although the relationships between ALDH1A1 expression and some clinicopathological parameters (tumor size, LNM, and the expression of HER2) was not definitive to some degree when we performed a subcategory analysis, the predictive values of ALDH1A1 expression for histological grade and survival of breast cancer patients were significant regardless of the different cutoff values of ALDH1A1 expression, the different districts where the patients were located, the different clinical stages of the patients, the difference in antibodies used in the studies, and the surgery status.

Conclusions: Our results indicate that ALDH1A1 is a biomarker to predict tumor progression and poor survival of breast cancer patients. This marker should be taken into consideration in the development of new diagnostic and therapeutic program for breast cancer.
\end{abstract}

Keywords: Breast cancer, Mammary cancer, Cancer stem cell, Aldehyde dehydrogenase 1 family member A1, Prognosis

\footnotetext{
* Correspondence: bianxiuwu@263.net; yushicang@163.com

${ }^{\dagger}$ Equal contributors

${ }^{1}$ Institute of Pathology and Southwest Cancer Center, Southwest Hospital,

Third Military Medical University, Chongqing 400037, China

${ }^{2}$ Key Laboratory of Tumor Immunology and Pathology of Ministry of

Education, Chongqing 400037, China

Full list of author information is available at the end of the article
} 


\section{Background}

Cancer stem cells (CSCs), although being a small percentage of the cancer cell population, are characterized by their multipotency and the ability to initiate cancer and propagate metastases [1-3]. Since the first report of these cells, which were found among acute myeloid leukemia cells by cell sorting technology using multiple surface markers [4], CSCs have been reported in various tumors, such as colon cancer [5], brain tumor [6], and lung cancer [7]. Due to their high tumorigenic and metastatic potential, CSCs are thought to be the most formidable obstacle to the successful treatment of cancer.

CSCs also have been isolated from breast cancer $[8,9]$, the most common malignancy in women worldwide. In 2003, Al-Hajj et al. have identified and isolated breast CSCs from patients using the cell surface marker pattern $\mathrm{CD}_{4} 4^{+} \mathrm{CD} 24^{- \text {llow }}$ Lineage ${ }^{-}[10]$. Subsequently, Ginestier et al. have reported that the activity of aldehyde dehydrogenase 1 (ALDH1) as assessed by the Aldefluor assay is a specific indicator for identifying, isolating, and tracking human breast CSCs [11].

The ALDH1A subfamily comprises three isoforms (ALDH1A1, ALDH1A2, and ALDH1A3), which synthesize retinoic acid (RA) from the retina and are crucial regulators for the RA signaling pathway. These enzymes have a high affinity for the oxidation of both all-trans- and 9-cisretinal and thereby serve to regulate the self-renewal and differentiation of normal stem cells and CSCs [12].

Although the exact isoform of ALDH1A responsible for the enzymatic activity assessed by BODIPY aminoacetaldehyde remains controversial [13-16], aldehyde dehydrogenase 1 family member A1 (ALDH1A1) is thought to have a predominant role [17]. Thus, much attention has been focused on the relationship between the expression of this isoform and the clinicopathologic parameters, including prognosis, of breast cancer patients.

However, the prognostic value of ALDH1A1 for breast cancer remains controversial despite numerous independent studies. For example, in a series of 577 breast carcinomas, Christophe Ginestier et al. demonstrated that ALDH1A1 expression detected by immunostaining correlated with poor patient prognosis [11]. Mieog et al. have revealed that the prognostic value of ALDH1A1 expression is age dependent and can be observed only in patients aged $<65$ years [18]. Using a retrospective collection of 321 node-negative and 318 node-positive breast cancer patients with a mean follow-up time of 12.6 years, Neumeister et al. found that ALDH1A1 expression alone does not significantly predict therapeutic outcome [19]. Therefore, we performed a systematic review and a meta-analysis to assess the robustness of the relationship between ALDH1A1 expression and clinicopathologic parameters/outcomes in breast cancer patients.

\section{Methods}

\section{Search strategy}

We conducted a search of the PubMed and EMBASE databases to identify studies for the systematic review. Two major groups of studies were created according to our objective. One group was used to clarify the association between ALDH1A1 expression and clinicopathological parameters, including tumor size, lymph node metastasis (LNM), histological grade, and the expression of growth factor receptors (estrogen receptor, ER; progesterone receptor, PR; epidermal growth factor receptor 2, HER2). The other group was used to investigate the association between ALDH1A1 expression and overall survival (OS)/disease-free survival (DFS).

The search terms were "ALDH1", "breast cancer". All studies were published prior to March 13, 2014. In the initial retrieved literatures, we read the titles or abstracts and screened for prognosis- and clinicopathology-related research. Studies were included when the following criteria were met: (1) published in English with the full text available, (2) the use of a case control design or a cohort design, and (3) the availability of data to allow the estimation of the hazard ratio (HR) for survival with a 95\% CI. Accordingly, the exclusion criteria were as follows: (1) reviews, abstracts and repeated studies; (2) ALDH1A1 not specified as the subtype expressed; and (3) the use of duplicate data. No ethnicity or regional restrictions were applied. The review process was performed by two independent reviewers.

\section{Data extraction}

The following information was extracted from these papers based the criteria listed above: first author, patients' country, publication year, research technique used, number of cases and controls, cutoff value for ALDH1A1, antibody used, type of tumor samples, and HR. For references that did not provide HRs, we referred to the methods described by Tierney et al. [20] to obtain the HRs using the data and figures from the original papers [19,21-23].

\section{Statistical analysis}

The prognosis of patients with breast cancer positive for ALDH1A1 expression was calculated using the unadjusted $\mathrm{HR}$ with the corresponding 95\% CI according the OS/specific survival (SS)/relative survival (RS) and DFS/metastasis-free survival (MFS)/recurrence-free survival (RFS) in cases and controls. We classified different prognostic parameters from included references, based on the characteristics of censored data, into two groups: (1) OS/SS/ RS; (2) DFS/MFS/RFS. Other clinicopathological factors were sorted into several subgroups: tumor size, LNM, histological grade, and the expression of ER, PR, and HER2. Fixed and random effects models were used to calculate a pooled odds ratio (OR) and HR. The statistical 
significance of the pooled OR and HR was evaluated with the $\mathrm{Z}$ test and $\mathrm{P}$ values, and $P<0.05$ was considered statistically significant. Heterogeneity across studies was evaluated by applying a $\mathrm{Q}$ test. In this approach, the $\mathrm{Q}$ value is defined as identical to the effect size of the $I^{2}$ value. A random effects model was used when the $I^{2}$ value for heterogeneity test was $>50 \%$; otherwise, a fixed effects model was used. Begg's rank correlation method and Egger's weighted regression method were used to assess publication bias $(P<0.05$ was considered statistically significant). All statistical tests for this meta-analysis were performed using STATA 11.0 software (STATA Corp., College Station, TX, USA).

\section{Results}

\section{Study characteristics}

A total of 16 studies from 15 publications [11,18,19,21-32] were found to meet the criteria for this analysis after the article titles, abstracts and main text were read to identify case reports and clinical outcomes. The flow chart for the identification of eligible studies is shown in Figure 1. The total number of patients was 3274, including 921 cases ALDH $1 \mathrm{Al}^{+}$breast cancer and a 2353 controls. Except in the study by Neumeister, immunohistochemistry (IHC) was a primary method used to evaluate ALDH1A1 expression in breast cancer specimens [19]. We identified the detected subtype as ALDH1A1 based on the antibodies listed in the references. For uniformed data analysis, tumor size T1 was considered as low stage, and T2, T3, and T4 as high stage. For the histological grade, all the studies used Nottingham Combined Histology Grade modified Scarff-Bloom-Richardson (SBR) grading system, grades I and II were grouped together $v s$. grade III. In the study by Ginestier et al., the patient samples were derived from two independent groups (America and France) [11]. Therefore, these samples were divided into two studies: the Ginestier U.M. set and the Ginestier I.P.C. set. The prognostic data from Lee et al. [26] was not available, because it was evaluated according to the change of expression of ALDH1A1 before and after the chemotherapy, rather than the categories ALDH1A1 ${ }^{+}$and ALDH1A1 ${ }^{-}$. The main characteristics of the 16 eligible studies are summarized in Table 1.

\section{Meta-analysis results Correlation of ALDH1A1 expression with clinicopathological parameters}

Overall analysis There were 14 references [11,18,21-32] that assessed ALDH1A1 expression and correlated it to tumor clinicopathological data. The overall analysis showed significant association between ALDH1A1 expression and tumor size, histological grade, LNM, and the expression of ER, PR, and HER2. Specifically, higher ALDH1A1 expression means greater tumor size, higher SBR grade, greater possibility of LNM, higher expression of HER2, and lower expression of ER and PR. The results are shown in Figure 2 and Table 2.

Subcategory analysis Subsequently, we performed a subcategory analysis according to different cutoff values of ALDH1A1 expression ( $>5 \%$ and $>0 \% / 1 \%$ subgroups),

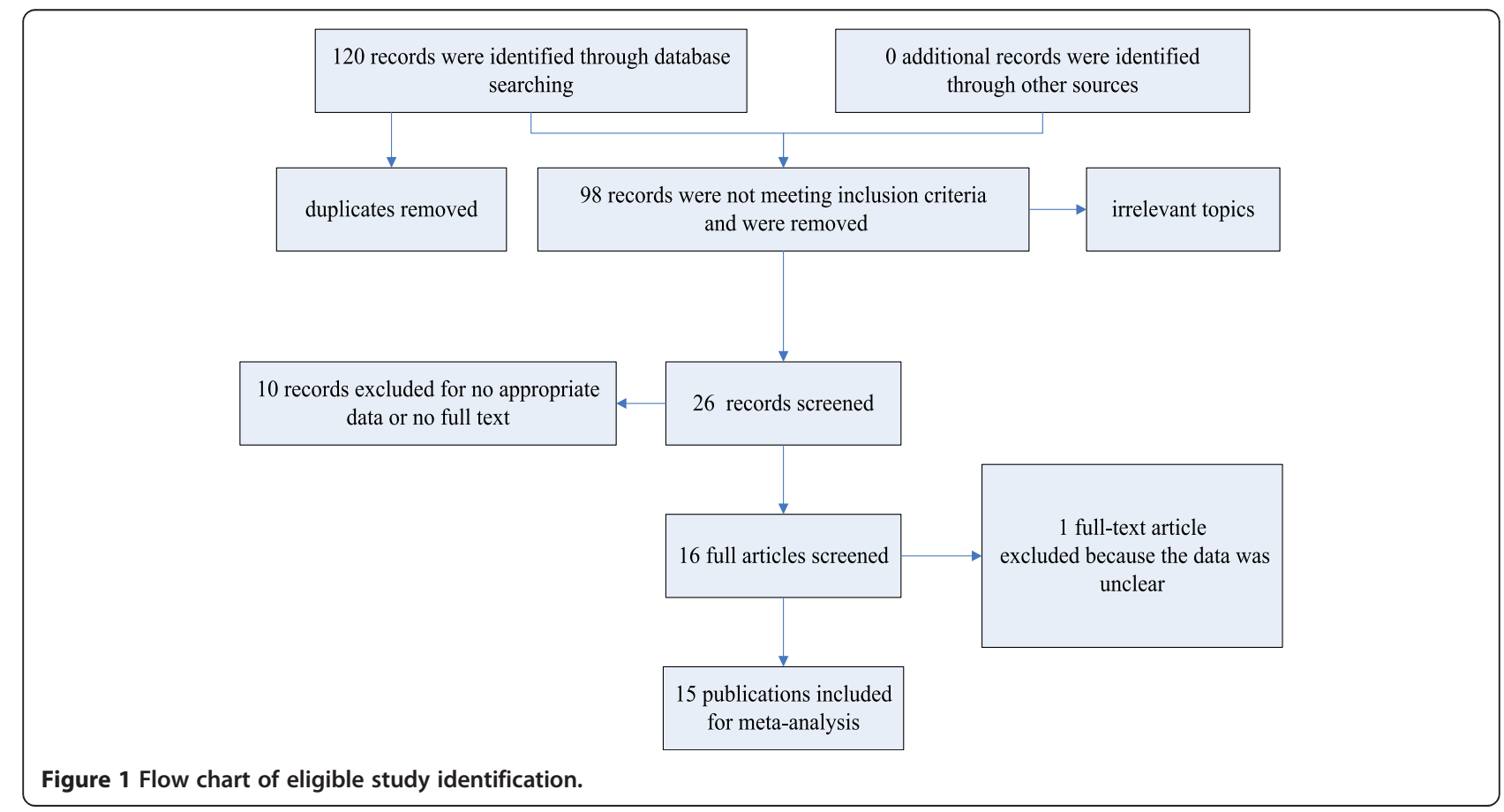


Table 1 Main characteristics of the eligible studies

\begin{tabular}{|c|c|c|c|c|c|c|c|c|c|}
\hline Author & Country & Year & Method & $\begin{array}{c}\text { Cases } \\
\left({\left.\mathrm{ALDH} 1 \mathrm{A1} 1^{+}\right)}\right.\end{array}$ & $\begin{array}{c}\text { Controls } \\
\left(\mathrm{ALDH1A1}^{-}\right)\end{array}$ & $\begin{array}{c}\text { Cutoff of } \\
\text { ALDH1A1 positive }\end{array}$ & Dilution of antibody & Situation of patients & HR \\
\hline Ginestier U.M. [1 1] & America & 2007 & $\mathrm{IHC}(\mathrm{TMA})$ & 24 & 122 & $>5 \%$ & BD Biosciences, 1:100 & NA & NA \\
\hline Ginestier I.P.C [11] & France & 2007 & $\mathrm{IHC}(\mathrm{TMA})$ & 102 & 243 & $>5 \%$ & BD Biosciences, 1:100 & NA & 1.76 \\
\hline Morimoto [27] & Japan & 2009 & $\mathrm{IHC}$ & 21 & 182 & $>0 \%$ & BD Biosciences, 1:100 & Treated with surgery & 1.516 \\
\hline Charafe-Jauffret [24] & France & 2010 & $\mathrm{IHC}$ & 29 & 53 & $>1 \%$ & BD Biosciences, 1:50 & $\begin{array}{l}\text { IBC, partly treated with } \\
\text { surgery }\end{array}$ & $\begin{array}{c}\text { SS, } 2.7 \\
\text { MFS, } 2.72\end{array}$ \\
\hline Erika Resetkova [29] & America & 2010 & IHC (TMA) & 35 & 159 & $>0 \%$ & BD Biosciences, 1:200 & Treated with surgery & NA \\
\hline Nalwoga [28] & Uganda & 2010 & IHC (TMA) & 88 & 95 & unclear & BD Biosciences, 1:250 & NA & NA \\
\hline Neumeister [19] & America & 2010 & $\begin{array}{l}\text { Immunofluorescent } \\
\text { assays (AQUA) }\end{array}$ & 45 & 581 & NA & BD Biosciences, 1:1000 & Treated with surgery & 2.32 \\
\hline Pei Yu [23] & China & 2010 & $\mathrm{IHC}$ & 18 & 78 & $>0 \%$ & Abcam, 1:100 & Treated with surgery & 4.6 \\
\hline He Lee [26] & Korea & 2011 & $\mathrm{IHC}$ & 12 & 80 & $>5 \%$ & BD Biosciences, 1:100 & $\begin{array}{c}\text { Stage }\|\sim\| I I \text {, treated with } \\
\text { surgery }\end{array}$ & 4.15 \\
\hline Yasuyo [31] & Japan & 2011 & $\mathrm{IHC}$ & 54 & 52 & $>0 \%$ & BD Biosciences, 1:1000 & TNBC, treated with surgery & 3.696 \\
\hline Yoshioka [32] & Japan & 2011 & $\mathrm{IHC}$ & 68 & 189 & $>0 \%$ & BD Biosciences, 1:1000 & Treated with surgery & $\begin{array}{c}\text { OS, } 1.93 \\
\text { RFS, } 1.667\end{array}$ \\
\hline Mieog [18] & Netherlands & 2012 & $\mathrm{IHC}$ & 292 & 195 & $>0 \%$ & BD Biosciences, NA & Treated with surgery & $\begin{array}{l}\text { RS, } 2.36 \\
\text { RFS, } 1.71\end{array}$ \\
\hline Nogami [21] & Japan & 2012 & $\mathrm{IHC}$ & 7 & 33 & $>5 \%$ & BD Biosciences, 1:200 & $\mathrm{ALNM}^{+}$, treated with surgery & 2.26 \\
\hline Sakakibara [22] & Japan & 2012 & $\mathrm{IHC}$ & 35 & 80 & $>5 \%$ & BD Biosciences, 1:200 & $\mathrm{ALNM}^{+}$, treated with surgery & 10.044 \\
\hline $\operatorname{Tan}[30]$ & $\begin{array}{l}\text { China, Malay, } \\
\text { Indian, Other }\end{array}$ & 2013 & $\mathrm{IHC}$ & 35 & 106 & $>10 \%$ & Abcam, 1:100 & Treated with surgery & NA \\
\hline Dong [25] & China & 2013 & $\mathrm{IHC}$ & 56 & 105 & $>5 \%$ & BD Biosciences, 1:200 & $\begin{array}{l}\text { Invasive ductal carcinoma and } \\
\text { ALNM }^{+} \text {, treated with surgery }\end{array}$ & $\begin{array}{l}\text { OS, } 3.309 \\
\text { RFS, } 2.774\end{array}$ \\
\hline
\end{tabular}

a) IBC was defined as inflammatory breast cancer, it is stage IIIB.

b) TMA was defined as tissue microarrays.

c) AQUA was defined as automated quantitative analysis.

d) TNBC breast cancer was defined as triple-negative breast cancer.

e) ALNM was defined as axillary lymph node metastases, it is $\geq$ Stage II. 


\section{A}

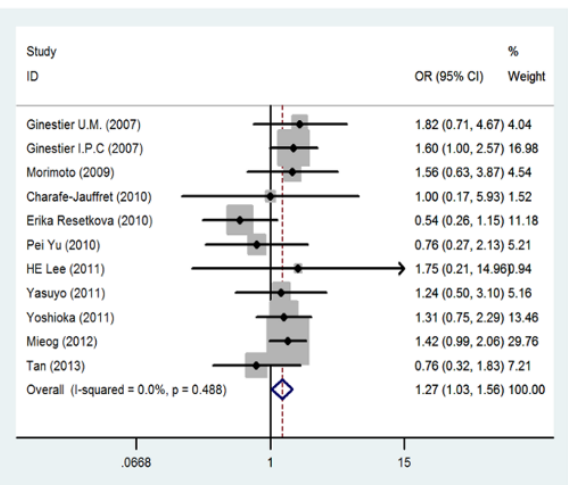

\section{C}

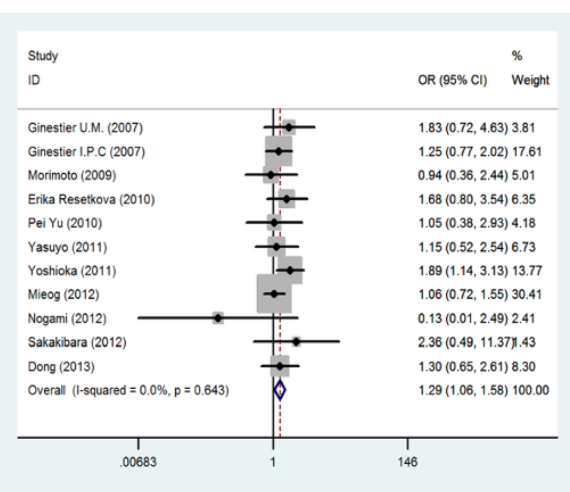

\section{E}

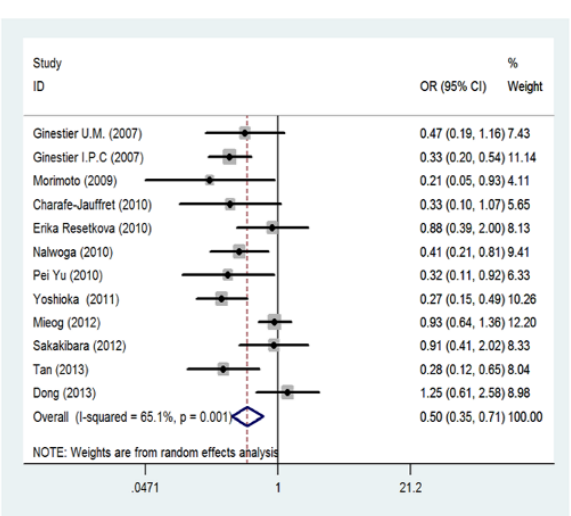

B

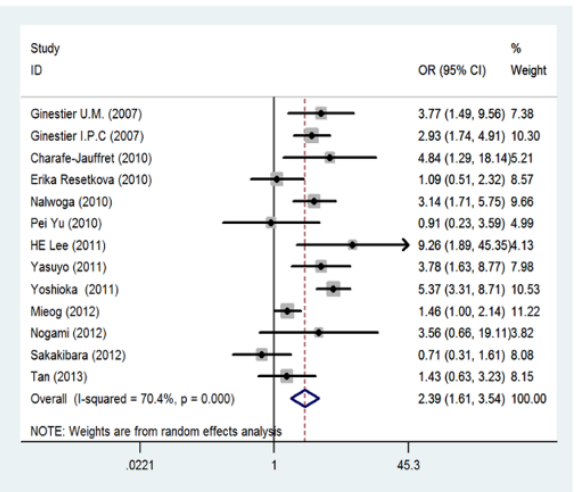

D

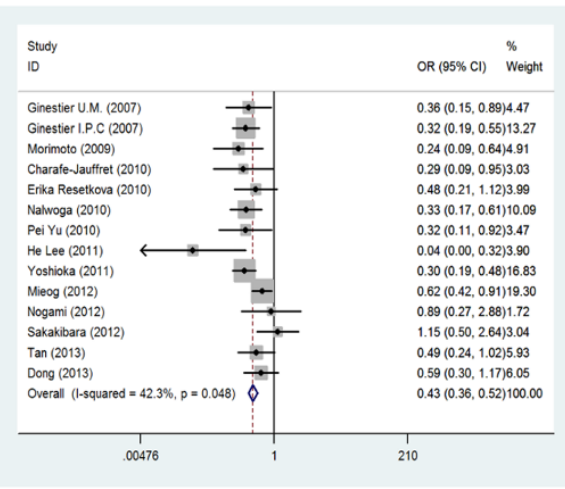

$\mathbf{F}$

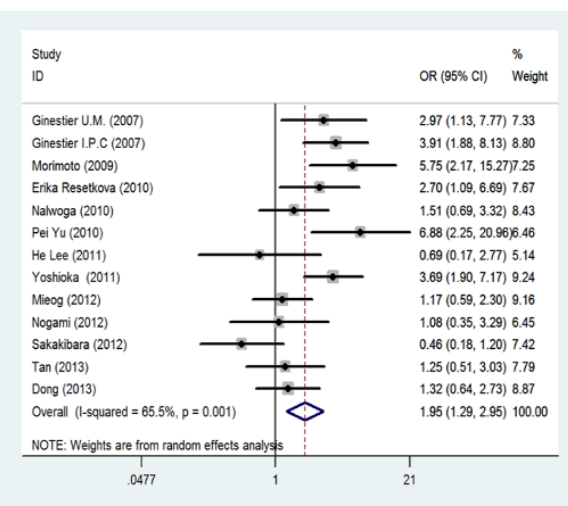

Figure 2 Meta-analysis of the association between ALDH1A1 expression and clinicopathological parameters: (A) LNM; (B) histological grade; (C) tumor size; (D) the expression of ER; (E) the expression of PR; (F) the expression of HER2.

different regions the patients originated from (AmericaEurope, Asia, and Africa subgroups), different clinical stages of the patients [No assesment (NA) and $\geq$ stage II subgroups], different antibodies used in the studies (BD subgroup and Abcam subgroup), and types of surgery for patients [Surgery, Part surgery, and No screened (NS) subgroups].
In the subgroup analysis based on the cutoff value, we found that ALDH1A1 expression is positively correlated with histological grade and negatively correlated with the expression of ER/PR, which is consistent with the results derived from overall analysis. At the same time, greater tumor size and higher expression of HER2 in the ALDH1A1 positive group could be found in the subgroup 
Table 2 Main results of meta-analysis according to the different cutoff values of ALDH1A1expression

\begin{tabular}{|c|c|c|c|c|c|c|c|c|c|c|c|c|}
\hline \multirow{3}{*}{ Parameter } & \multicolumn{4}{|c|}{ Cutoff value } & \multicolumn{4}{|c|}{ Patients' district } & \multicolumn{4}{|c|}{ Patients' stage } \\
\hline & \multicolumn{2}{|c|}{$5 \%$} & \multicolumn{2}{|c|}{$0 \%$ or $1 \%$} & \multicolumn{2}{|c|}{ America-Europe } & \multicolumn{2}{|c|}{ Asia } & \multicolumn{2}{|c|}{ NA } & \multicolumn{2}{|c|}{$\geq$ Stage II } \\
\hline & OR/HR & $95 \% \mathrm{Cl}$ & OR/HR & $95 \% \mathrm{Cl}$ & OR/HR & $95 \% \mathrm{Cl}$ & OR/HR & $95 \% \mathrm{Cl}$ & OR/HR & $95 \% \mathrm{Cl}$ & OR/HR & $95 \% \mathrm{Cl}$ \\
\hline Tumor size & 1.30 & $0.92-1.83$ & $1.29^{*}$ & $1.01-1.65$ & 1.24 & $0.95-1.61$ & $1.37^{*}$ & $1.01-1.86$ & $1.31^{*}$ & $1.06-1.62$ & 1.19 & $0.66-2.14$ \\
\hline LNM & 1.43 & $0.99-2.07$ & 1.20 & $0.94-1.54$ & $1.33^{*}$ & $1.03-1.72$ & 1.16 & $0.82-1.64$ & $1.27^{*}$ & $1.03-1.56$ & 1.29 & $0.33-4.97$ \\
\hline SBR grade & $2.33^{*}$ & $1.22-4.43$ & $2.32^{*}$ & $1.20-4.49$ & $2.16^{*}$ & $1.31-3.55$ & $2.41^{*}$ & $1.16-5.04$ & $2.37^{*}$ & $1.57-3.58$ & 2.94 & $0.80-10.77$ \\
\hline ER & $0.46^{*}$ & $0.34-0.61$ & $0.43^{*}$ & $0.33-0.55$ & $0.47^{*}$ & $0.36-0.61$ & $0.42^{*}$ & $0.32-0.54$ & $0.41^{*}$ & $0.33-0.50$ & $0.54^{*}$ & $0.36-0.82$ \\
\hline PR & $0.54^{*}$ & $0.30-0.98$ & $0.46^{*}$ & $0.25-0.84$ & $0.56^{*}$ & $0.33-0.96$ & $0.45^{*}$ & $0.24-.0 .84$ & $0.43^{*}$ & $0.29-0.64$ & 0.82 & $0.42-1.61$ \\
\hline HER2 & 1.39 & $0.78-2.48$ & $3.19^{*}$ & $1.67-6.08$ & $2.39^{*}$ & $1.33-4.28$ & 1.78 & $0.93-3.42$ & $2.66^{*}$ & $1.76-4.03$ & 0.89 & $0.54-1.48$ \\
\hline OS/SS/RS & $2.65^{*}$ & $1.82-3.86$ & $2.33^{*}$ & $1.60-3.38$ & $2.39^{*}$ & $1.83-3.13$ & $3.10^{*}$ & $2.02-4.75$ & $2.25^{*}$ & $1.71-2.95$ & $3.51^{*}$ & $2.33-5.31$ \\
\hline \multirow[t]{2}{*}{ DFS/MFS/RFS } & $2.65^{*}$ & $1.54-4.57$ & $2.04^{*}$ & $1.53-2.73$ & $1.95^{*}$ & $1.33-2.85$ & $2.36^{*}$ & $1.67-3.32$ & $1.93^{*}$ & $1.41-2.65$ & $2.68^{*}$ & $1.73-4.13$ \\
\hline & \multicolumn{4}{|c|}{ Antibodies used in studies } & \multicolumn{4}{|c|}{ Surgery situation of patients } & \multirow{2}{*}{\multicolumn{4}{|c|}{ Overall }} \\
\hline \multirow[t]{2}{*}{ Parameter } & \multicolumn{2}{|c|}{ BD } & \multicolumn{2}{|c|}{ Abcam } & \multicolumn{2}{|c|}{ Surgery } & \multicolumn{2}{|c|}{ NS/Partly } & & & & \\
\hline & OR/HR & $95 \% \mathrm{Cl}$ & OR/HR & $95 \% \mathrm{Cl}$ & OR/HR & $95 \% \mathrm{Cl}$ & OR/HR & $95 \% \mathrm{Cl}$ & OR/HR & $95 \% \mathrm{Cl}$ & $P$ & $1^{2}$ \\
\hline Tumor size & $1.30^{*}$ & $1.06-1.60$ & 1.05 & $0.38-2.93$ & - & - & - & - & $1.29^{*}$ & $1.06-1.58$ & 0.012 & $0.0 \%$ \\
\hline LNM & $1.34^{*}$ & $1.08-1.67$ & 0.76 & $0.39-1.48$ & - & - & - & - & $1.27^{*}$ & $1.03-1.56$ & 0.024 & $0.0 \%$ \\
\hline SBR grade & $2.65^{*}$ & $1.73-4.08$ & 1.27 & $0.63-2.56$ & - & - & - & - & $2.39^{*}$ & $1.61-3.54$ & 0.000 & $70.4 \%$ \\
\hline ER & $0.43^{*}$ & $0.35-0.52$ & $0.43^{*}$ & $0.36-0.52$ & - & - & - & - & $0.43^{*}$ & $0.36-0.52$ & 0.000 & $42.3 \%$ \\
\hline PR & $0.54^{*}$ & $0.36-0.80$ & $0.30^{*}$ & $0.15-0.57$ & - & - & - & - & $0.50^{*}$ & $0.35-0.71$ & 0.000 & $65.1 \%$ \\
\hline HER2 & $1.85^{*}$ & $1.19-2.87$ & 2.83 & $0.53-15.07$ & - & - & - & - & $1.95^{*}$ & $1.29-2.95$ & 0.002 & $65.5 \%$ \\
\hline OS/SS/RS & - & - & - & - & $2.87^{*}$ & $2.17-3.80$ & $1.76^{*}$ & 1.06-2.91- & $2.58^{*}$ & $2.05-3.23$ & 0.000 & $38.0 \%$ \\
\hline DFS/MFS/RFS & $2.07^{*}$ & $1.60-2.69$ & $4.60^{*}$ & $1.53-13.81$ & $2.09^{*}$ & $1.60-2.75$ & $2.72^{*}$ & $1.32-5.60$ & $2.16^{*}$ & $1.68-2.79$ & 0.000 & $0.0 \%$ \\
\hline
\end{tabular}

Ps. *means a significant difference.

studies with cutoff values $>0 \%$ or $1 \%$. However, LNM status is not correlated with ALDH1A1 expression regardless of cutoff value (Table 2 and Additional file 1: Figure S1).

Because there was only one study for African patients, meta-analysis was performed for the America-Europe and Asia subcategories according to different regions of the patients. We found that the relationship between ALDH1A1 expression and histological grade or the expression of ER/PR is the same as the results from previous overall analysis, regardless of regions of origin. However, tumor size in the America-Europe subgroup is not related to ALDH1A1 expression. In addition, greater possibility of LNM and higher expression of HER2 could be found in America-Europe patients with high ALDH1A1 expression in tumor (Table 2 and Additional file 2: Figure S2).

For subcategory analysis based on the clinical stage, six clinicopathological parameters are all correlated with ALDH1A1 expression in the NA group. However, in the group $\geq$ stage II, ALDH1A1 expression is only correlated with ER expression (Table 2 and Additional file 3: Figure S3).

For subcategory analysis based on the antibodies, six clinicopathological parameters are also correlated with ALDH1A1 expression in the BD group. In the Abcam group, ALDH1A1 expression is only correlated with the expression of ER and PR (Table 2 and Additional file 4: Figure S4).
Impact of ALDH1A1 expression on survival for breast cancer There were a total of 11 references [11,18,19,21-25, $27,31,32]$ relating to the association between ALDH1A1 expression and breast cancer prognosis. The prognosis was evaluated by the indicators OS/SS/RS and DFS/MFS/RFS. The studies by Charafe-Jauffret [24], Yoshioka [32] and Mieog [18] used two types of prognosis indicators, which were classified by characteristics; OS/SS/RS made up one group, DFS/MFS/RFS made up the other group.

Overall analysis The data for this analysis indicated that the prognosis of breast cancer patients with ALDH1A1 $1^{+}$ was poorer than that of the ALDH1A1 $1^{-}$patients regardless of the indicators used (OS/SS/RS or DFS/MFS/RFS). The results were shown as follows: OS/SS/RS: OR $=2.58$, 95\% CI $=2.05-3.23, P=0.000, I^{2}=38.0 \%$; DFS $/$ MFS $/$ RFS: $\mathrm{OR}=2.16, \quad 95 \% \quad \mathrm{CI}=1.68-2.79, \quad P=0.000, \quad I^{2}=0.0 \%$ (Figure 3).

Subcategory analysis $\mathrm{ALDH} 1 \mathrm{~A} 1^{+}$breast cancer patients have poorer prognosis in all subcategory analysis. The results are shown in Table 2, Figure 3 and Additional file 5: Figures S5, Additional file 6: Figure S6, Additional file 7: Figure S7, Additional file 8: Figure S8 and Additional file 9: Figure S9. 
Sensitivity analysis Sensitivity analysis was performed through the sequential omission of individual studies. The corresponding pooled OR was not altered significantly for any study factor after sequentially excluding each study, demonstrating that our data are stable and reliable.

\section{Publication bias}

Begg's funnel plot and Egger's test were used to evaluate the publication bias of all the relevant literature. The statistical results did not show evidence of publication bias: tumor size: Begg's test, $P=0.755$, Egger's test, $P=$ 0.721; LNM: Begg's test, $P=0.640$, Egger's test, $P=0.342$; histological grade: Begg's test, $P=0.583$, Egger's test, $P=$ 0.766; expression of ER: Begg's test, $P=0.511$, Egger's test, $P=0.360$; expression of PR: Begg's test, $P=0.537$, Egger's test, $P=0.278$; expression of HER2: Begg's test, $P=0.855$, Egger's test, $P=0.749$. Similar results were found for OS/SS/RS: Begg's test, $P=0.368$, Egger's test, $P=0.155$; DFS/MFS/RFS: Begg's test, $P=0.266$, Egger's test, $P=0.169$. The funnel plot used to investigate the relationship between ALDH1A1 expression and tumor size is shown in Figure 4. The shape of the funnel plot did not show obvious evidence of asymmetry.

\section{Discussion}

It is well known that ALDH1A1 can be used as a marker for breast CSCs, which have high tumor-initiating and self-renewal capabilities. Because of the important role performed by breast CSCs in tumorigenesis, development, and therapeutic outcomes, many groups have investigated the relationship between the expression of ALDH1A1 and the clinicopathologic features of breast cancer patients. However, there are discrepancies among the studies attempted to assess the association. Our results derived from the meta-analysis of existing studies indicated that ALDH1A1 can be used as a poor prognostic indicator in breast cancer patients. The high expression of ALDH1A1 is positively associated with larger tumor size, higher histological grade and a greater likelihood of LNM in breast cancer patients. In addition, the expression of ALDH1A1 was positively correlated with the expression of HER2 but negatively correlated with the expression of ER/PR. Moreover, if we performed subcategory analysis based on the different cutoff values of ALDH1A1 expression, the different regions of origin of the patients, the different clinical stages of the patients selected, and the different antibodies used in studies, the relationships between ALDH1A1 expression and some clinicopathological parameters, including tumor size, LNM, and the expression of HER2, are slightly different. For example, the positive correlation between ALDH1A1 expression and the tumor size only could be found in the cutoff $>0 / 1 \%$, Asia, NA, and BD subgroups. Regarding LNM, a significantly positive relationship with ALDH1A1 expression presented in the America-Europe, NA, and BD subgroups. In addition, the positive relationship between ALDH1A1 and HER2 expression was observed in the cutoff $>0 / 1 \%$, America-Europe, NA, and BD subgroups.

Only one eligible study from Yoshioka et al. indicated that ALDH1A1 expression was significantly correlated

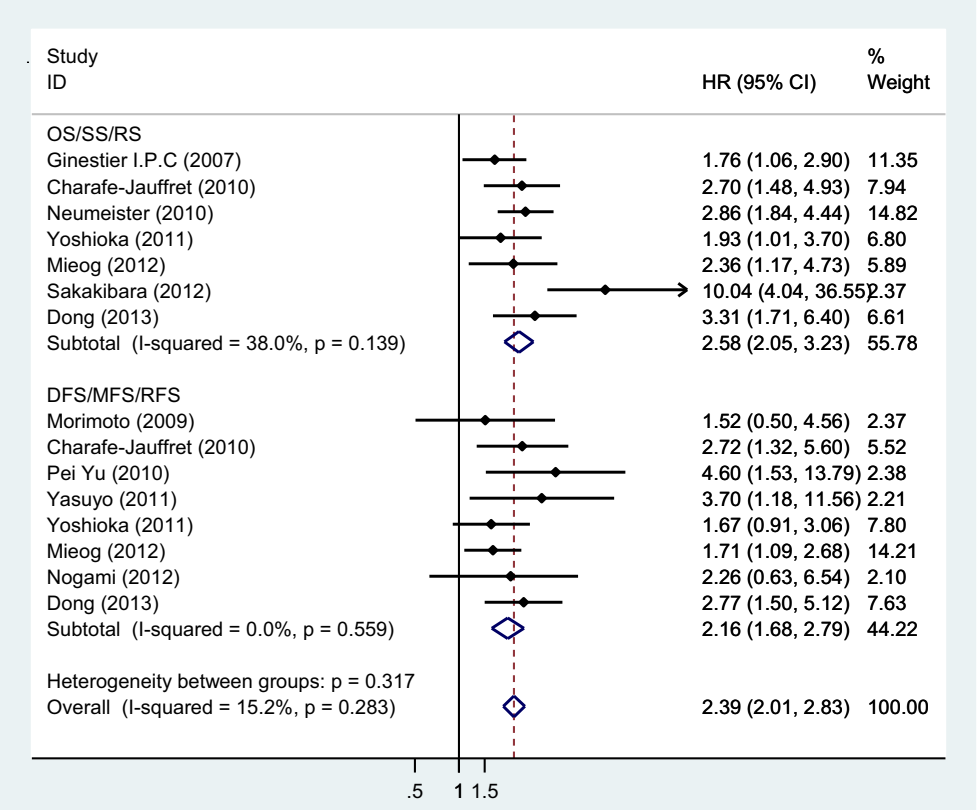

Figure 3 Meta-analysis of the association between ALDH1A1 expression and prognosis, including OS/SS/RS and DFS/MFS/RFS. 


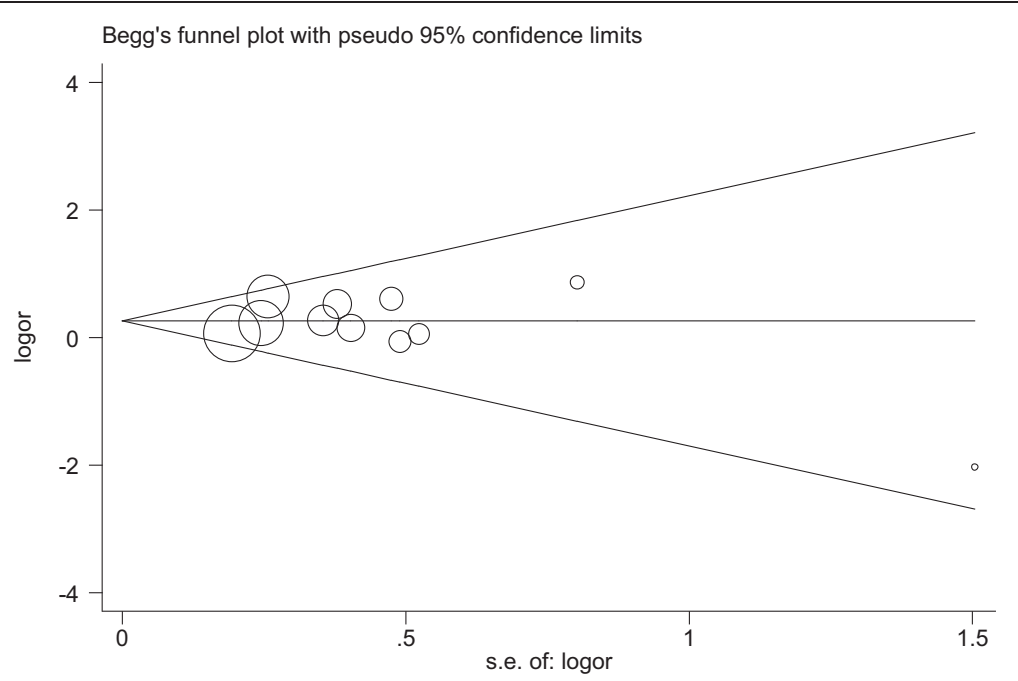

Figure 4 Begg's funnel plot of publication bias. Each point represents a separate study for the indicated association (Tumor size).

with larger tumor size $(>2.0 \mathrm{~cm})$ [32]. However, our results revealed that high expression of ALDH1A1 correlated with larger tumor size, especially in the cutoff $>0 / 1 \%$, Asia, $\mathrm{NA}$, and BD subgroups. Multicenter prospective studies based on large, homogeneous patient populations will be required to assess the relationship between tumor size and ALDH1A1 expression.

None of the studies eligible for the meta-analysis indicated that ALDH1A1 expression was correlated with LNM. However, our results from larger samples revealed that there is a significant positive association between these two parameters, especially in the America-Europe, NA, and BD subgroups. This is supported by another study by Neumeister $e t$ al. that was not included in our meta-analysis due to the lack of some required informations. The study indicated that there is a significant association between ALDH1A1 and LNM (OR $=2.37 ; 95 \%$ $\mathrm{CI}=1.582-3.165)$ [19]. In addition, a significant correlation between ALDH1A1 expression in the primary tumor and in the corresponding metastatic lymph nodes has been observed. In a group of 48 breast cancer samples with LNM, Yu et al. found that there were $8 \mathrm{ALDH}^{\mathrm{A} 1^{+}}$samples among the primary cancer tissues and 7 positive samples among the corresponding lymph node tissues. In addition, there were $40 \mathrm{ALDH} 1 \mathrm{~A} 1^{-}$samples among the primary cancer tissues, and 39 negative cases among the corresponding lymph node tissues $(P<0.05)$ [23]. Similar results were also observed by Nogami [21]. These results suggest that ALDH1A1 might have an important role in LNM, and this relationship was manifested in the results of our meta-analysis. However, there was no significant correlation found between ALDH1A1 expression and LNM in the Asia, zstage II, and Abcam subgroups. This indicated that the previous controversial conclusions about ALDH1A1 expression and
LNM might result from the different races, clinical stages, and antibodies used in studies; however, there are only 2 studies using the antibody from Abcam, which might reduce the power and accuracy of subcategory analysis. In addition, there is no significant correlation between ALDH1A1 expression and the 5 clinicopathological parameters (tumor size, LNM, SBR grade, PR, and HER2) in the $\geq$ stage II subgroup. The small number of included studies might also be the reason for this situation. At the same time, it suggests that using the expression level of a single molecule to assess the disease development of advanced breast cancer patients might be inadequate.

Based on the expression patterns of different molecular markers, breast cancer can be divided into more than six similar subgroups, which have distinguishing features with respect to clinical outcomes, responses to adjuvant therapy, and patterns of metastatic recurrence [33,34]. In addition, a recent study suggested that there is a close relationship between the subtypes defined by gene expression profiling and the cellular origin of breast cancer $[35,36]$. Thus, we also want to know the relationship between ALDH1A1 expression and the three most important molecular markers of breast cancer, ER, PR, and HER2. The results derived from overall analysis suggested that the overexpression of ALDH1A1 might be related to the enriched-HER2 subtype of breast cancer $\left(\mathrm{ER}^{-} \mathrm{PR}^{-} \mathrm{HER} 2^{+}\right)$, which is derived from the transformation of mammary late luminal progenitor cells $[35,36]$. However, it should be noted that: First, the positive correlation between ALDH1A1 expression and HER2 is only observed in the America-Europe subgroup. Second, there were discrepancies regarding the definition of HER2 positivity in the different studies. In some studies, tumors with scores of $2+$ and $3+$ were considered to be HER2 positive (more than 
$10 \%$ of the cells showed positive immunohistochemical staining) $[11,23,27]$. In other studies, only tumors with scores of $3+$ were considered HER2 positive (more than $30 \%$ of the cells showed positive immunohistochemical staining) $[21,22,26,32]$. Only three studies confirmed the amplification of HER2 by fluorescence in-situ hybridization analysis [22,26,29]. Thus, other subtypes defined by gene expression profiling, such as basal-like breast cancer with moderate expression of HER2 $(2+\sim 3+)$, might have been included in the $\mathrm{HER}^{+}$group in this meta-analysis. ALDH1A1 expression might also be related to some basallike breast cancers, which are derived from the transformation of mammary luminal progenitor cells [35,36]. The results of Nalwoga et al. confirmed this possibility. They found that there was a close relationship between ALDH1A1 expression and the HER2 subtype $(\mathrm{OR}=3.6$, $95 \% \mathrm{CI}=1.4-9.7)$ and the basal-like subtype $(\mathrm{OR}=4.0$, $95 \% \mathrm{CI}=1.8-8.8$ ) [28]. Similar results were found in the study presented by Lee [26]. These data suggest that ALDH1A1 could be used as a potential therapeutic target for breast cancers of the HER2-enriched subtype or partial basal-like subtype, especially in patients derived from America-Europe.

It should be noted that there are some limitations to this meta-analysis. First, although we endeavored to extract valid data from survival curves, in which HRs were not directly measured, these indirect data are less reliable than direct data from the original literature because these calculated HRs are the result of univariate analyses and might contain some deviations. Second, all of the studies included in our meta-analyses are retrospective. Their experimental design may contribute to the heterogeneity, which might reduce the analysis power to some extent. Therefore, larger multicenter prospective studies based on homogeneous populations are required to validate the prognostic power of ALDH1A1. Third, publication bias is a concern. We tried to identify all relevant data, but some data were still missing. Some missing information, such as the results presented by Marcato et al. [16], might reduce the power of ALDH1A1 as a prognostic predictor in breast cancer patients.

\section{Conclusion}

This meta-analysis indicates that ALDH1A1 is an important predictor of the progression and poor survival of breast cancer patients. Our results suggest that the analysis of ALDH1A1 expression in breast cancer not only provides a better understanding of the relationship between breast tumorigenesis and cancer genomics but may also be beneficial for the design of treatment and the assessment of the prognosis of patients. We will further study the influence of ALDH1A1 expression on differentiation, invasion, and metastasis of breast cancer cells.

\section{Additional files}

Additional file 1: Figure S1. Meta-analysis of the association between ALDH1A1 expression and clinicopathological parameters according to the cutoff value of ALDH1A1 expression: (A) LNM; (B) histological grade; (C) tumor size; (D) the expression of ER; $(\mathbf{E})$ the expression of PR; $(\mathbf{F})$ the expression of HER2.

Additional file 2: Figure S2. Meta-analysis of the association between ALDH1A1 expression and clinicopathological parameters according to the regions of origin of patients: $(\mathbf{A}) \mathrm{LNM}$; $(\mathbf{B})$ histological grade; $(\mathbf{C})$ tumor size; (D) the expression of ER; (E) the expression of PR; (F) the expression of HER2.

Additional file 3: Figure S3. Meta-analysis of the association between ALDH1A1 expression and clinicopathological parameters according to the stage of patients: (A) LNM; (B) histological grade; $(\mathbf{C})$ tumor size; (D) the expression of $\mathrm{ER} ;(\mathbf{E})$ the expression of PR; $(\mathbf{F})$ the expression of HER2.

Additional file 4: Figure S4. Meta-analysis of the association between ALDH1A1 expression and clinicopathological parameters according to the different antibodies used in the studies: (A) LNM; (B) histological grade; $(\mathbf{C})$ tumor size; (D) the expression of ER; (E) the expression of PR; (F) the expression of HER2.

Additional file 5: Figure S5. Meta-analysis of the association between ALDH1A1 expression and the prognosis according to the regions of origin of patients: (A) OS/SS/RS; (B) DFS/MFS/RFS.

Additional file 6: Figure S6. Meta-analysis of the association between ALDH1A1 expression and the prognosis according to the stage of patients: (A) OS/SS/RS; (B) DFS/MFS/RFS.

Additional file 7: Figure S7. Meta-analysis of the association between ALDH1A1 expression and the prognosis according to the different antibodies used in the studies (DFS/MFS/RFS).

Additional file 8: Figure S8. Meta-analysis of the association between ALDH1A1 expression and the prognosis according to the surgery situation of patients: (A) OS/SS/RS; (B) DFS/MFS/RFS.

Additional file 9: Figure S9. Meta-analysis of the association between ALDH1A1 expression and the prognosis according to the cutoff value of ALDH1A1 expression: (A) OS/SS/RS; (B) DFS/MFS/RFS.

\section{Abbreviations}

ALDH1A1: Aldehyde dehydrogenase 1 family member A1; HER2: Epidermal growth factor receptor 2; CSC: Cancer stem cell; ALDH1: Aldehyde dehydrogenase 1; LNM: Lymph node metastasis; ER: Estrogen receptor; PR: Progesterone receptor; OS: Overall survival; DFS: Disease-free survival; HR: Hazard ratio; OR: Odd ratio; SS: Specific survival; RS: Relative survival; MFS: Metastasis-free survival; RFS: Recurrence-free survival; IHC: Immunohistochemistry; IBC: Inflammatory breast cancer; TMA: Tissue microarrays; AQUA: Automated quantitative analysis; TBNC: Triple-negative breast cancer; ALNM: Axillary lymph node metastases; NA: No assessment; NS: No screened.

\section{Competing interests}

The authors declare no conflict of interest.

\section{Authors' contributions}

$Y L$ and DL helped to design the overall study, compile and curate the datasets, design the statistical approaches, perform the computational analysis, and develop the biological interpretation. $Y L$ and $D L$ contributed equally to this work. JD and SX provided expertise in clinical breast oncology. JZ and XY helped to design the statistical approaches and perform the computational analysis. $Y C$ and $X Z$ helped to design the overall study and design the statistical approaches. SY and XB designed the overall study, compiled and curated the datasets, designed the statistical approaches, performed the computational analysis, developed biological interpretation, and wrote the manuscript. All authors contributed to the preparation of the manuscript and read and approved the final version. 


\section{Acknowledgments}

We would like to thank Dr. Yan-qi Zhang, and Dr. Chuan Xu for their constructive suggestions. This study was supported by grants from the National Natural Science Foundation of China (No. 81172071), Outstanding Youth Science Foundation of Chongqing (No. CSTC2013JCYJJQ1003), and National Basic Research Program of China (973 Program, No. 2010CB529400).

\section{Author details}

'Institute of Pathology and Southwest Cancer Center, Southwest Hospital, Third Military Medical University, Chongqing 400037, China. ${ }^{2}$ Key Laboratory of Tumor Immunology and Pathology of Ministry of Education, Chongqing 400037, China. ${ }^{3}$ School of Biomedical Sciences, The Chinese University of Hong Kong, Hongkong, China.

Received: 18 November 2013 Accepted: 6 June 2014 Published: 17 June 2014

\section{References}

1. Gupta PB, Chaffer $C L$, Weinberg RA: Cancer stem cells: mirage or reality? Nat Med 2009, 15(9):1010-1012.

2. Polyak K, Hahn WC: Roots and stems: stem cells in cancer. Nat Med 2006 12(3):296-300

3. Zhao D, Najbauer J, Annala AJ, Garcia E, Metz MZ, Gutova M, Polewski MD, Gilchrist M, Glackin CA, Kim SU, Aboody KS: Human neural stem cell tropism to metastatic breast cancer. Stem Cells 2012, 30(2):314-325.

4. Lapidot T, Sirard C, Vormoor J, Murdoch B, Hoang T, Caceres-Cortes J, Minden M, Paterson B, Caligiuri MA, Dick JE: A cell initiating human acute myeloid leukaemia after transplantation into SCID mice. Nature 1994, 367(6464):645-648

5. Bourseau-Guilmain E, Griveau A, Benoit JP, Garcion E: The importance of the stem cell marker prominin-1/CD133 in the uptake of transferrin and in iron metabolism in human colon cancer Caco-2 cells. PLOS One 2011, 6(9):e25515.

6. Dirks PB: Brain tumor stem cells: the cancer stem cell hypothesis writ large. Mol Oncol 2010, 4(5):420-430.

7. Wang P, Gao Q, Suo Z, Munthe E, Solberg S, Ma L, Wang M, Westerdaal NA, Kvalheim G, Gaudernack G: Identification and characterization of cells with cancer stem cell properties in human primary lung cancer cell lines. PLoS One 2013, 8(3):e57020.

8. Harrison H, Rogerson L, Gregson HJ, Brennan KR, Clarke RB, Landberg G: Contrasting hypoxic effects on breast cancer stem cell hierarchy is dependent on ER-alpha status. Cancer Res 2013, 73(4):1420-1433.

9. Korkaya H, Kim Gl, Davis A, Malik F, Henry NL, Ithimakin S, Quraishi AA, Tawakkol N, D'Angelo R, Paulson AK, Chung S, Luther T, Paholak HJ, Liu S, Hassan KA, Zen Q, Clouthier SG, Wicha MS: Activation of an IL6 inflammatory loop mediates trastuzumab resistance in HER2+ breast cancer by expanding the cancer stem cell population. Mol Cell 2012, 47 (4):570-584.

10. Al-Hajj M, Wicha MS, Benito-Hernandez A, Morrison SJ, Clarke MF: Prospective identification of tumorigenic breast cancer cells. Proc Natl Acad Sci U S A 2003, 100(7):3983-3988.

11. Ginestier $\mathrm{C}$, Hur MH, Charafe-Jauffret $E$, Monville F, Dutcher J, Brown M, Jacquemier J, Viens P, Kleer CG, Liu S, et al: ALDH1 is a marker of normal and malignant human mammary stem cells and a predictor of poor clinical outcome. Cell Stem Cell 2007, 1(5):555-567.

12. Koppaka V, Thompson DC, Chen Y, Ellermann M, Nicolaou KC, Juvonen RO, Petersen D, Deitrich RA, Hurley TD, Vasiliou V: Aldehyde dehydrogenase inhibitors: a comprehensive review of the pharmacology, mechanism of action, substrate specificity, and clinical application. Pharmacol Rev 2012 64(3):520-539

13. Eirew $P$, Kannan N, Knapp DJ, Vaillant F, Emerman JT, Lindeman GJ, Visvader $\mathrm{JE}$, Eaves $\mathrm{CJ}$ : Aldehyde dehydrogenase activity is a biomarker of primitive normal human mammary luminal cells. Stem Cells 2012, 30(2):344-348.

14. Luo Y, Dallaglio K, Chen Y, Robinson WA, Robinson SE, McCarter MD, Wang J, Gonzalez R, Thompson DC, Norris DA, Roop DR, Vasiliou V, Fujita M: ALDH1A isozymes are markers of human melanoma stem cells and potential therapeutic targets. Stem Cells 2012, 30(10):2100-2113.

15. Mao P, Joshi K, Li J, Kim SH, Li P, Santana-Santos L, Luthra S, Chandran UR, Benos PV, Smith L, Wang M, Hu B, Cheng SY, Sobol RW, Nakano I: Mesenchymal glioma stem cells are maintained by activated glycolytic metabolism involving aldehyde dehydrogenase 1A3. Proc Natl Acad SCi U S A 2013, 110(21):8644-8649.

16. Marcato $P$, Dean CA, Pan D, Araslanova R, Gillis M, Joshi M, Helyer L, Pan L, Leidal A, Gujar S, Giacomantonio CA, Lee PW, Giacomantonio CA, Lee PW: Aldehyde dehydrogenase activity of breast cancer stem cells is primarily due to isoform ALDH1A3 and its expression is predictive of metastasis. Stem Cells 2011, 29(1):32-45.

17. Marcato P, Dean CA, Giacomantonio CA, Lee PW: Aldehyde dehydrogenase: its role as a cancer stem cell marker comes down to the specific isoform. Cell Cycle 2011, 10(9):1378-1384.

18. Mieog JS, de Kruijf EM, Bastiaannet E, Kuppen PJ, Sajet A, de Craen AJ, Smit $V T$, van de Velde CJ, Liefers GJ: Age determines the prognostic role of the cancer stem cell marker aldehyde dehydrogenase- 1 in breast cancer. BMC Cancer 2012, 12:42.

19. Neumeister $V_{\text {, Agarwal }}$, Bordeaux J, Camp RL, Rimm DL: In situ identification of putative cancer stem cells by multiplexing ALDH1, CD44, and cytokeratin identifies breast cancer patients with poor prognosis. Am J Pathol 2010, 176(5):2131-2138.

20. Tierney JF, Stewart LA, Ghersi D, Burdett S, Sydes MR: Practical methods for incorporating summary time-to-event data into meta-analysis. Trials 2007, 8:16

21. Nogami T, Shien T, Tanaka T, Nishiyama K, Mizoo T, Iwamto T, Ikeda H, Taira $\mathrm{N}$, Doihara $\mathrm{H}$, Miyoshi S: Expression of ALDH1 in axillary lymph node metastases is a prognostic factor of poor clinical outcome in breast cancer patients with 1-3 lymph node metastases. Breast Cancer 2012, 21 (1):58-65.

22. Sakakibara M, Fujimori T, Miyoshi T, Nagashima T, Fujimoto H, Suzuki HT, Ohki Y, Fushimi K, Yokomizo J, Nakatani Y, Miyazaki M: Aldehyde dehydrogenase 1-positive cells in axillary lymph node metastases after chemotherapy as a prognostic factor in patients with lymph node-positive breast cancer. Cancer 2012, 118(16):3899-3910.

23. YU P, Zhou LWJ, Jiang AF, Li K: Prognostic relevance of ALDH1 in breast cancer: a clinicopathological study of 96 cases. Chin-German J Clin Oncol 2010, 9:31-35

24. Charafe-Jauffret E, Ginestier C, lovino F, Tarpin C, Diebel M, Esterni B, Houvenaeghel G, Extra JM, Bertucci F, Jacquemier J, Xerri L, Dontu G, Stassi G, Xiao Y, Barsky SH, Birnbaum D, Viens P, Wicha MS: Aldehyde dehydrogenase 1-positive cancer stem cells mediate metastasis and poor clinical outcome in inflammatory breast cancer. Clin Cancer Res 2010, 16(1):45-55.

25. Dong Y, Bi LR, Xu N, Yang HM, Zhang HT, Ding Y, Shi AP, Fan ZM: The expression of aldehyde dehydrogenase 1 in invasive primary breast tumors and axillary lymph node metastases is associated with poor clinical prognosis. Pathol Res Pract 2013, 209(9):555-561.

26. Lee HE, Kim JH, Kim YJ, Choi SY, Kim SW, Kang E, Chung IY, Kim IA, Kim EJ, Choi Y, Ryu HS, Park SY: An increase in cancer stem cell population after primary systemic therapy is a poor prognostic factor in breast cancer. Br J Cancer 2011, 104(11):1730-1738.

27. Morimoto K, Kim SJ, Tanei T, Shimazu K, Tanji Y, Taguchi T, Tamaki Y, Terada N, Noguchi S: Stem cell marker aldehyde dehydrogenase 1-positive breast cancers are characterized by negative estrogen receptor, positive human epidermal growth factor receptor type 2, and high Ki67 expression. Cancer Sci 2009, 100(6):1062-1068.

28. Nalwoga $H$, Arnes JB, Wabinga $H$, Akslen LA: Expression of aldehyde dehydrogenase 1 (ALDH1) is associated with basal-like markers and features of aggressive tumours in African breast cancer. Br J Cancer 2010, 102(2):369-375.

29. Resetkova E, Reis-Filho JS, Jain RK, Mehta R, Thorat MA, Nakshatri H, Badve S: Prognostic impact of ALDH1 in breast cancer: a story of stem cells and tumor microenvironment. Breast Cancer Res Treat 2010, 123(1):97-108.

30. Tan EY, Thike AA, Tan PH: ALDH1 expression is enriched in breast cancers arising in young women but does not predict outcome. Br J Cancer 2013, 109(1):109-113.

31. Yasuyo O, Umekita Y, Yoshioka T, Souda M, Rai Y, Sagara Y, Sagara Y, Sagara $Y$, Tanimoto A: Aldehyde dehydrogenase 1 expression predicts poor prognosis in triple-negative breast cancer. Histopathology 2011, 59(4):776-780.

32. Yoshioka T, Umekita Y, Ohi Y, Souda M, Sagara Y, Rai Y, Tanimoto A: Aldehyde dehydrogenase 1 expression is a predictor of poor prognosis in node-positive breast cancers: a long-term follow-up study. Histopathology 2011, 58(4):608-616. 
33. Neve RM, Chin K, Fridlyand J, Yeh J, Baehner FL, Fevr T, Clark L, Bayani N, Coppe JP, Tong F, Speed T, Spellman PT, DeVries S, Lapuk A, Wang NJ, Kuo WL, Stilwell JL, Pinkel D, Albertson DG, Waldman FM, McCormick F, Dickson RB, Johnson MD, Lippman M, Ethier S, Gazdar A, Gray JW: A collection of breast cancer cell lines for the study of functionally distinct cancer subtypes. Cancer Cell 2006, 10(6):515-527.

34. Subik K, Lee JF, Baxter L, Strzepek T, Costello D, Crowley P, Xing L, Hung MC, Bonfiglio T, Hicks DG, Tang P: The Expression Patterns of ER, PR, HER2, CK5/6, EGFR, Ki-67 and AR by Immunohistochemical Analysis in Breast Cancer Cell Lines. Breast Cancer 2010, 4:35-41.

35. Lim E, Vaillant F, Wu D, Forrest NC, Pal B, Hart AH, Asselin-Labat ML, Gyorki DE, Ward T, Partanen A, Feleppa F, Huschtscha LI, Thorne HJ, KConFab, Fox SB, Yan M, French JD, Brown MA, Smyth GK, Visvader JE, Lindeman GJ: Aberrant luminal progenitors as the candidate target population for basal tumor development in BRCA1 mutation carriers. Nat Med 2009, 15(8):907-913.

36. Prat A, Perou CM: Mammary development meets cancer genomics. Nat Med 2009, 15(8):842-844.

doi:10.1186/1471-2407-14-444

Cite this article as: Liu et al.: ALDH1A1 expression correlates with

clinicopathologic features and poor prognosis of breast cancer patients: a systematic review and meta-analysis. BMC Cancer 2014 14:444.

\section{Submit your next manuscript to BioMed Central and take full advantage of:}

- Convenient online submission

- Thorough peer review

- No space constraints or color figure charges

- Immediate publication on acceptance

- Inclusion in PubMed, CAS, Scopus and Google Scholar

- Research which is freely available for redistribution 